\title{
COMO AS CRIANÇAS DE 7 A 11 ANOS EXPLICAM SUA CEFALÉIA
}

\author{
Rosana M.R. Mendonça' ${ }^{1}$, Yára D. Fragoso², Ricardo Diniz ${ }^{3}$
}

\begin{abstract}
RESUMO - O objetivo deste estudo foi observar a capacidade de crianças de 7 a 11 anos de idade em descrever sua cefaléia na anamnese. Para tanto, foram feitas duas avaliações de 94 crianças em entrevistas individuais pela mesma pediatra, com intervalo de seis a oito semanas, sem a presença de adultos. As características da dor de cabeça destas crianças puderam ser avaliadas nestas entrevistas, não havendo dados significativamente conflitantes entre as duas sessões. Embora habitualmente descrita e valorizada pelo adulto que acompanha a consulta, a cefaléia da infância deveria ser informada pelo próprio paciente. Neste grupo de crianças de 7 a 11 anos as informações foram obtidas sem dificuldade quando se permitiu à criança que usasse suas próprias palavras, no tempo que fosse necessário.
\end{abstract}

PALAVRAS-CHAVE: cefaléia, enxaqueca, infância.

\section{How children aged 7 to 11 explain their headache}

ABSTRACT - The aim of the present study was to observe the ability of children aged 7 to 11 in describing their headache during anamnesis. For this purpose, two evaluations of 94 children were performed in individual assessments, done by the same Pediatrician, within a six to eight week interval, without the presence of adults. The characteristics of headache in these children could be properly evaluated during the interviews. There were no remarkable conflicting information between the two interviews. Although the headache of the child is usually described by the accompanying adult during the consultation, childhood headache should really be informed by the patient. In this group of young children (7 to 11 years old), information could be obtained without difficulty since we allowed the child enough time and the use of his (her) own words.

KEY WORDS: headache, migraine, childhood.

Estima-se que até $40 \%$ das crianças brasileiras sofram de cefaléia ${ }^{1}$. Os estudos de cefaléia na infância são raros², muitos deles com falhas metodológicas, tendendo a supervalorizar a informação do adulto acompanhante em detrimento da informação da criança ${ }^{3}$. Os autores acreditam que as crianças são capazes de explicar os detalhes de suas crises de dor e que os achados de tais avaliações são confiáveis.

Crianças com cefaléia costumam ser consultadas pelo pediatra e não pelo neurologista ${ }^{3}$. Os autores consideram que os pediatras devem conhecer bem as nuances do diagnóstico das cefaléias, uma vez que farão o atendimento primário destes pacientes.

O presente estudo foi proposto com a finalidade de determinar como crianças de 7 a 11 anos podem descrever características da própria cefaléia durante a anamnese.

\section{MÉTODO}

O protocolo deste estudo foi avaliado e aprovado pelo Comitê de Ética em Pesquisa da Faculdade de Medicina da UNIMES. Todas as crianças participantes deste estudo tiveram autorização escrita dos pais ou responsável, estando estes cientes do caráter investigativo deste projeto. Não foram realizados exames médicos ou laboratoriais. As crianças foram encorajadas a conversar com pais ou responsável sobre as entrevistas e sobre os resultados das avaliações.

Duas escolas particulares da cidade de Santos, SP, concordaram em participar deste estudo. Em visitas às classes de alunos da $1^{\text {a }}$ à $6^{a}$ série do ensino fundamental, foi solicitado às crianças que apresentassem cefaléia que se identificassem. A estas crianças foi dado um formulário explicando o objetivo e métodos do estudo, para que discutissem com os pais ou responsável. Estando estes de acordo, os formulários eram retornados preenchidos e assinados. A criança era então entrevistada individualmente pela pediatra, visando a caracterização da cefaléia sem

Faculdade de Medicina da UNIMES, Santos SP, Brasil: ${ }^{1}$ Pediatra responsável pelas avaliações das crianças; ${ }^{2}$ Professora Titular de Neurologia Clínica da Faculdade de Medicina da UNIMES; ${ }^{3}$ Professor Titular de Clínica Médica da Faculdade de Medicina da UNIMES.

Recebido 29 Setembro 2003, recebido na forma final 27 Fevereiro 2004. Aceito 27 Março 2004.

Dr. Ricardo Diniz - Rua da Constituição 374 - 11015-470 Santos SP - Brasil. E-mail: diretoria.medicina@unimes.com.br 
a influência de acompanhantes adultos. As entrevistas foram feitas em local calmo, sendo permitido que a criança escrevesse, desenhasse e usasse suas próprias palavras para descrever a cefaléia. Não havia limite de tempo para responder ao questionário durante a entrevista, sendo permitida a livre expressão da criança. Opções de respostas às características da dor somente foram apresentadas à criança quando exemplos eram solicitados por ela, e ainda assim sempre com duas ou três possibilidades.

Na primeira consulta os seguintes dados foram obtidos: tempo de duração da cefaléia; freqüência de crises; localização da dor; caráter da dor; duração da dor; intensidade da dor, fatores de melhora; fatores de piora; sintomas acompanhantes da dor; antecedentes familiares de cefaléia; médicos consultados.

Após a primeira avaliação, foi solicitado à criança que prestasse atenção em todos os dados acima relacionados, pois este questionário seria respondido novamente depois de algumas semanas. Havendo alguma crise de cefaléia no intervalo das duas avaliações, a criança deveria observar com cuidado os detalhes e características desta crise. Não houve registro em diário detalhado, porém foi encorajado o registro e anotações pela criança entre as duas entrevistas.
Após seis a oito semanas, as crianças foram todas reavaliadas pela mesma pediatra, sob as mesmas condições, com o mesmo questionário.

\section{RESULTADOS}

Um grupo de 94 crianças, uniformemente distribuído para sexo e idade, foi entrevistado em duas ocasiões pela mesma pediatra (R.M.). A Tabela 1 mostra um resumo dos dados demográficos deste grupo.

A Tabela 2 mostra os resultados da caracterização da cefaléia de acordo com a idade e também no grupo todo. Sessenta e cinco crianças $(69,1 \%)$ apresentavam cefaléia há mais de um ano; quatro diziam mesmo ter dor "desde que nasceram". A

Tabela 1. Dados demográficos das 94 crianças participantes deste estudo.

\begin{tabular}{ccccccc}
\hline & 7 anos & 8 anos & 9 anos & 10 anos & 11 anos & Total \\
\hline Meninos & 6 & 8 & 10 & 10 & 10 & 44 \\
Meninas & 10 & 10 & 10 & 10 & 10 & 50 \\
\hline
\end{tabular}

Tabela 2. Caracterização da cefaléia nas diferentes faixas etárias $(n=94)$.

\begin{tabular}{|c|c|c|c|c|c|c|}
\hline & 7 anos & 8 anos & 9 anos & 10 anos & 11 anos & Total \\
\hline \multicolumn{7}{|l|}{ Tempo de história de cefaléia } \\
\hline$<6$ meses & 1 & 0 & 3 & 4 & 0 & 8 \\
\hline 6 meses a 1 ano & 6 & 2 & 3 & 4 & 6 & 21 \\
\hline$>1$ ano & 9 & 16 & 14 & 12 & 14 & 65 \\
\hline \multicolumn{7}{|l|}{ Freqüência de crises * } \\
\hline$<1$ por semana & $9(7)$ & $3(9)$ & $8(6)$ & $8(9)$ & $0(4)$ & $28(35)$ \\
\hline 1 a 2 por semana & $4(6)$ & $10(6)$ & $5(9)$ & $8(6)$ & $12(5)$ & $39(32)$ \\
\hline Pelo menos 15 dias/mês & $2(0)$ & $5(3)$ & $2(3)$ & $4(3)$ & $4(8)$ & $17(17)$ \\
\hline Rara (< 1 por mês) & $1(3)$ & $0(0)$ & $5(2)$ & $0(2)$ & $4(3)$ & $10(10)$ \\
\hline \multicolumn{7}{|l|}{ Localização da dor } \\
\hline Bifrontal ("testa") & 8 & 10 & 14 & 9 & 8 & 49 \\
\hline Temporal & 4 & 3 & 0 & 7 & 7 & 21 \\
\hline Holocraneana & 4 & 5 & 6 & 3 & 4 & 22 \\
\hline Não sabe / varia muito & 0 & 0 & 0 & 1 & 1 & 2 \\
\hline Tendência a unilateralidade & 5 & 3 & 3 & 6 & 5 & 22 \\
\hline \multicolumn{7}{|l|}{ Tipo de dor } \\
\hline Aperto & 3 & 5 & 5 & 4 & 5 & 22 \\
\hline Peso & 6 & 7 & 5 & 5 & 2 & 25 \\
\hline Latejante & 7 & 4 & 9 & 9 & 13 & 42 \\
\hline Outra / não sabe & 0 & 2 & 1 & 2 & 0 & 5 \\
\hline \multicolumn{7}{|l|}{ Duração da dor } \\
\hline$<1$ hora & 6 & 9 & 8 & 4 & 9 & 36 \\
\hline 1 a 4 horas & 7 & 4 & 9 & 14 & 6 & 40 \\
\hline$>4$ horas & 3 & 5 & 3 & 2 & 3 & 16 \\
\hline Não sabe / varia muito & 0 & 0 & 0 & 0 & 2 & 2 \\
\hline \multicolumn{7}{|l|}{ Intensidade da dor } \\
\hline Fraca & 2 & 1 & 3 & 1 & 4 & 11 \\
\hline Moderada & 11 & 9 & 10 & 16 & 9 & 55 \\
\hline Forte & 3 & 8 & 7 & 3 & 7 & 28 \\
\hline
\end{tabular}

* valores entre parênteses se referem à $2^{\mathrm{a}}$ entrevista. 
Tabela 3. Fatores acompanhantes, fatores de melhora e de piora da cefaléia nas diferentes faixas etárias $(n=94)$.

\begin{tabular}{|c|c|c|c|c|c|c|}
\hline & 7 anos & 8 anos & 9 anos & 10 anos & 11 anos & Total \\
\hline \multicolumn{7}{|l|}{ Fatores acompanhantes } \\
\hline Alterações visuais & 6 & 7 & 12 & 9 & 10 & 44 \\
\hline Náusea e/ou vômito & 6 & 2 & 4 & 6 & 3 & 21 \\
\hline Tontura & 4 & 2 & 2 & 4 & 8 & 20 \\
\hline \multicolumn{7}{|l|}{ Fatores de piora da dor } \\
\hline Luz, claridade, sol & 10 & 9 & 12 & 7 & 8 & 46 \\
\hline Som, barulho & 7 & 6 & 8 & 9 & 6 & 36 \\
\hline Cheiro & 3 & 10 & 2 & 8 & 2 & 25 \\
\hline Esforço, cansaço & 1 & 0 & 2 & 3 & 1 & 7 \\
\hline Medo/nervoso & 1 & 1 & 0 & 5 & 3 & 10 \\
\hline Fome & 0 & 3 & 6 & 6 & 6 & 21 \\
\hline Choro & 0 & 6 & 2 & 0 & 0 & 8 \\
\hline \multicolumn{7}{|l|}{ Fatores de melhora da dor } \\
\hline Repouso & 9 & 8 & 11 & 11 & 12 & 51 \\
\hline Sono & 3 & 7 & 9 & 9 & 9 & 37 \\
\hline Medicação & 4 & 11 & 12 & 12 & 6 & 45 \\
\hline \multicolumn{7}{|l|}{ Intensidade da dor } \\
\hline Fraca & 2 & 1 & 3 & 1 & 4 & 11 \\
\hline Moderada & 11 & 9 & 10 & 16 & 9 & 55 \\
\hline Forte & 3 & 8 & 7 & 3 & 7 & 28 \\
\hline
\end{tabular}

localização da dor foi predominantemente frontal; $52 \%$ das crianças a referiam como "na testa", espalmando a mão sobre a região. Em 22 crianças $(23,4 \%)$ observou-se tendência a unilateralidade da dor nas crises. O caráter latejante da dor predominou entre os entrevistados, sendo referida por 42 crianças $(44,7 \%)$. Dor em peso $(26,6 \%)$ e aperto $(23,4 \%)$ também foram freqüentes.

A duração da dor foi menor ou igual a 4 horas em $88,9 \%$ das crianças, havendo predomínio de dor moderada $(58,5 \%)$ ou forte $(29,8 \%)$. Repouso e sono foram freqüentemente citados como fatores de melhora (93,2\%), embora seja preocupante o número de crianças que fazem uso de medicação analgésica $(47,9 \%)$. Entre os fatores de piora da dor, destacaram-se luz $(48,9 \%)$, som $(38,3 \%)$ e odores (26,6\%). Estes dados estão detalhados na Tabela 3.

É necessário observar que $46,8 \%$ das crianças relataram algum tipo de alteração visual durante a crise, descrevendo estrelinhas, luzes, escurecimento da visão, visão embaçada, dificuldade para ler ou pontos escuros no campo visual. Tais descrições não puderam ser caracterizadas como auras visuais das crises.

A convivência com dores de cabeça era observada em $83 \%$ das crianças que referiam cefaléia em pelo menos um parente em primeiro grau.

Deste grupo de 94 crianças, 39 (41,5\%) já haviam sido consultadas por médico devido à cefaléia. Apenas duas crianças haviam sido avaliadas por neurologistas, tendo as demais sido consultadas por pe- diatras, otorrinolaringologistas, homeopatas e oftalmologistas.

As informações obtidas na segunda entrevista não trouxeram dados significativamente diferentes, exceto pela freqüência de crises, fato observado também em adultos na prática clínica diária.

\section{DISCUSSÃO}

A cuidadosa e detalhada história da cefaléia é fundamental para o diagnóstico tanto em adultos quanto em crianças ${ }^{4}$. Apenas com o diagnóstico correto será possível instituir o tratamento adequado ${ }^{5}$, inclusive evitando o uso abusivo de analgésicos que poderá mudar a evolução natural da cefaléia primária ${ }^{6}$. No presente estudo, as crianças entrevistadas foram capazes de detalhar as características de sua cefaléia de forma bastante satisfatória, assertiva, e com segurança. As crianças responderam sem dificuldade as questões formuladas e deram exemplos, fizeram comparações, se esforçando para tornar tudo muito claro. Por exemplo, quando solicitadas a descrever o caráter da dor, as crianças explicavam que "era como um coração batendo", ou "era como uma coisa muito pesada em cima da cabeça". A capacidade em descrever claramente os detalhes da dor não estava restrita aos mais velhos. As 94 crianças de 7 a 11 anos de idade responderam objetivamente e, exceto pela freqüência de crises, os dados das duas entrevistas foram bastante semelhantes. Frente a casos de simulação e de dados inconsistentes, seria esperado que as respostas fossem pe- 
lo menos em parte contraditórias após intervalo de pelo menos seis semanas.

Não tivemos a intenção de diagnosticar a cefaléia destas crianças, apenas avaliamos o papel da criança na anamnese. No entanto, devido ao tempo de dor, freqüência de crises, intensidade e duração da cefaléia, fatores de piora e de melhora, e fatores acompanhantes, nos parece que boa parte desta população seria portadora de enxaqueca ${ }^{7}$. Resultados semelhantes foram obtidos por outros autores trabalhando com populações brasileiras ${ }^{8,9}$.

Nossa sugestão aos pediatras seria que a criança com cefaléia pudesse descrever a própria dor. $\mathrm{O}$ adulto acompanhante poderá não valorizar os mesmos dados que a criança que sofre da dor, sendo mesmo muito difícil a alguém descrever a dor de outrem. Obviamente, como citado por outros pesquisadores, pais e filhos podem concordar em vários itens relativos à cefaléia, e ambos devem ser ouvidos $^{10}$.

Embora a enxaqueca na infância seja freqüente ${ }^{11}$ é comum que ela seja sub-diagnosticada em crianças $^{12}$. É raro que o neurologista esteja envolvido em avaliações iniciais de cefaléia na infância ${ }^{3}$ e, nas clínicas de atendimento de cefaléia, existe claro predomínio das formas já crônicas ${ }^{13,14}$. O caráter crônico da cefaléia estava presente em 18,1\% das crianças de nosso estudo, devido à freqüência maior ou igual a 15 dias por mês com cefaléia.

O não-especialista consultado para avaliar uma criança com cefaléia pode obter muitos dados relevantes na primeira consulta. Em entrevistas subseqüentes, as crianças podem ter reconsiderado os detalhes da dor e informar ainda melhor sobre as crises. Diários de registro de cefaléia podem auxiliar nestas avaliações ${ }^{15-17}$ e devem ser encorajados. Podem ser utilizados diários detalhados, padronizados pelos serviços de atendimento em cefaléia, porém a simples anotação pela criança e familiares pode trazer informações importantes nas primeiras consultas.

\section{REFERÊNCIAS}

1. Nunes ML, Gherpelli JLD. Diagnóstico e tratamento em neurologia infantil [Editorial]. J Pediatr (Rio J) 2002;78(Supl 1):S1.

2. Gherpelli JLD. Tratamento das cefaléias. J Pediatr (Rio J) 2002;78(Supl 1):S3-S8.

3. Fragoso YD, Ferreira DM, Cavinato AB, Tsutida CY, Rodrigues S. O papel do oftalmologista na consulta da criança com cefaléia. Rev Bras Oftalmol 1997;56:316-321.

4. Lewis DW. Headaches in children and adolescents. Am Fam Physician 2002;65:625-632.

5. McGrath PA. Chronic daily headache in children and adolescents. Curr Pain Headache Rep 2001;5:557-566.

6. Mathew NT. Transformed migraine, analgesic rebound, and other chronic daily headaches. Neurol Clin 1997;15:167-186.

7. IHS - International Headache Society. Headache Classification Committee classification and diagnostic criteria for headache disorders, cranial neuralgias and facial Pain. Cephalalgia 1988;8(Suppl 7):S1-S96.

8. Fontenelle MLC, Cwajg S. Enxaqueca na infância: dificuldades diagnósticas. Arq Neuropsiquiatr 1998;56:553-558.

9. Barea LM, Tannhauser M, Rotta NT. An epidemiologic study of headache among children and adolescents of southern Brazil. Cephalalgia 1996;16:545-549.

10. Antoniuk S, Kozak MF, ML, Michelon L, Montemor MR Netto. Prevalence of headache in children of a school from Curitiba, Brazil, comparing data obtained from children and parents. Arq Neuropsiquiatr, 1998;56:726-733.

11. Rothner AD. Headaches in children and adolescents: update 2001. Semin Pediatr Neurol 2001;8:2-6.

12. Wasiewski WW, Rothner DA. Pediatric migraine headache diagnosis, evaluation and management. Neurologist 1999;5:122-134.

13. Koenig MA, Gladstein J, McCarter RJ, Hershey AD, Wasiewski W. Chronic daily headache in children and adolescents presenting to tertiary headache clinics. Headache 2002;42:491-500.

14. Winner P, Gladstein J. Chronic daily headache in pediatric practice. Curr Opin Neurol 2002;15:297-301.

15. Metsahonkala L, Sillanpaa M, Tuominen J. Headache diary in the diagnosis of childhood migraine. Headache 1997;37:240-244.

16. Nielsen KD, Rasmussen C, Russell MB. The diagnostic headache diary: a headache expert system. Stud Health Technol Inform 2000;78:149-160.

17. van den Brink M, Bandell-Hoekstra EN, Abu-Saad HH. The occurrence of recall bias in pediatric headache: a comparison of questionnaire and diary data. Headache 2001;41:11. 